\title{
HÁBITOS ALIMENTICIOS DEL JUREL Trachurus murphyi (Nichols) EN LA ZONA NOR-CENTRAL DEL MAR PERUANO
}

\section{JACK MACKEREL Trachurus murphyi (Nichols) FEEDING HABITS IN THE NORTH-CENTRAL PERUVIAN SEA}

\author{
Henry Orrego ${ }^{1}$ y Jaime Mendo ${ }^{2}$
}

\begin{abstract}
Resumen
Trachurus murphyi es una de las especies más importantes de la red trófica del ecosistema pelágico-oceánico y representa una de las pesquerías industriales de mayor significancia de la costa del Perú. Con la finalidad de conocer el comportamiento dietario de esta especie se analizaron los contenidos estomacales de 3175 ejemplares capturados a bordo de embarcaciones industriales arrastreras semi-pelágicas y cerqueras en la zona norte y centro del Perú respectivamente desde 1995 hasta 1997. La longitud total del jurel fluctuó entre 22 y 59 cm y mayormente en un rango de 26 y $46 \mathrm{~cm}$. Estadísticamente se comprueba que la variación observada de los contenidos estomacales es significativa para todo el periodo de estudio como también para los diferentes grupos de tallas. El porcentaje de estómagos con alimento en las diferentes clases de longitud fluctuaron entre 42.3 y 100\%. Los crustáceos contribuyeron con el $60.4 \%$ de la masa de alimento ingerido, seguido por los peces con 39.3\%, mientras que los moluscos sólo representaron el $0.36 \%$. Dentro de estos grupos la especie más representativa es Euphausia mucronata (54.3\%) y peces (35.5\%) pero sin poder identificar la especie predominante por el estado de descomposición en los contenidos estomacales. En el año 1997 (El Niño) se produce un aumento considerable en el número de especies como presas llegando a 23 especies. Aunque con pequeñas variantes en cada año se destaca la preferencia de eufausidos para los ejemplares más pequeños $(<38 \mathrm{~cm})$ y de peces $(>42 \mathrm{~cm})$. Basados en el IRI y el Indice de Jerarquización se destaca la notable preferencia de los eufausidos como presa, esencialmente la $E$. mucronata en los años 1995 y 1996 pero diversificándose en 1997 con otras especies de eufausidos y de peces en su dieta, manifestándose su carácter de predador oportunista. El análisis cluster para los datos de contenido estomacal para el año 1995 organizó las 8 clases de longitud en 2 grupos principales de tallas de $<26$ a $49.9 \mathrm{~cm}$ y un grupo representado por la clase de 50 a 53.9 cm. En el año 1996 se observa algo muy similar y en 1997 un año con evento de El Niño, los individuos forman dos grupos definidos en relación al contenido estomacal, un grupo de individuos entre $<26$ a $53.9 \mathrm{~cm}$ y el otro entre 54 a $>58 \mathrm{~cm}$.
\end{abstract}

Palabras clave: Trachurus murphyi, dieta alimenticia, El Niño, Perú.

\section{Abstract}

Trachurus murphyi is one of the most relevant species in the food chains of the pelagicoceanic system and represents one of the most important industrial fisheries in the Peruvian coast. In order to know its predatory behavior, stomach contents of 3175 individuals were analyzed. The individuals were caught on board in industrial semi-pelagic trawlers and purse seine vessels in the north and central coast of Peru from 1995 to 1997. Individuals' total lengths ranged from 22 to 58 $\mathrm{cm}$, the main range being from 26 to $46 \mathrm{~cm}$. Percentage of stomachs with food of different length classes ranged from 42.3 to $100 \%$. Crustaceans were the largest item in the diet with $60.4 \%$ followed by fishes with $39.3 \%$ and mollusks with only $0.36 \%$. Euphausia. mucronata was the most representative species in the diet with $54.3 \%$ followed by a $35.5 \%$ of fishes which could not be identified due to decomposition processes. Based on IRI values, the predominant groups in the diet corresponded to the Eufausidae family. Cluster analysis applied to the stomachs contents sampled in 1995 by length classes organized the individuals into 2 groups: <26 a $49.9 \mathrm{~cm}$ and 50$53.9 \mathrm{~cm}$. For 1996 it is similar to 1995 and for 1997 a year affected by an El Niño event, cluster analysis grouped the jack mackerel stomachs of into two well defined groups $<26$ to $53.9 \mathrm{~cm}$ and 54 to $>58 \mathrm{~cm}$.

Key words: Trachurus murphyi, feeding habits, El Niño, Peru. 


\section{Introducción.}

Especies del género Trachurus (Perciformes, Carangidae) son muy comunes y están ampliamente distribuidas en diferentes áreas de la costa del Océano Pacífico. El Jurel Trachurus murphyi es una especie cuya distribución geográfica abarca principalmente el Océano Pacífico Suroriental (frente a la costa sudamericana) y, secundariamente, el Océano Pacífico Suroccidental, al sur de Nueva Zelandia (Subsecretaría de Pesca, 2000). Su distribución abarca desde el norte del Perú hasta la zona central-sur de Chile (Berry \& Cohen, 1974). Batimétricamente, se distribuye entre 10 y $180 \mathrm{~m}$, llegando en ocasiones a $300 \mathrm{~m}$ de profundidad (Pastor, 1994).

En la costa peruana el jurel representa una pesquería pelágica muy importante, estando destinada su captura principalmente para el consumo humano directo. En el año 2009 el desembarque de jurel fue de 25912 toneladas con mayores rendimientos en el primer trimestre y una biomasa estimada en 70074 toneladas, mientras que en el 2013 el desembarque fue de aproximadamente de 28241 toneladas (Imarpe, 2009, 2013); siendo el desembarque del 2013 el 85 \% inferior al promedio histórico. De acuerdo a los desembarques desde 1970 hasta la actualidad los años 1995, 1996 y 1997 presentaron desembarques anuales superiores a 350 mil toneladas, con un máximo en 1997 superior a las 600 mil toneladas representando el segundo máximo histórico después del desembarque del año 2001.

La literatura disponible en el Perú sobre el recurso jurel es variada en relación a su biología, distribución, abundancia y patrones de comportamiento ambiental pudiéndose resaltar algunos de los estudios recientes (Imarpe, 2009, 2013; Sánchez et al., 2013; Dioses, 2013, Flores et al., 2013; Espino, 2013, Ñiquen et al., 2013) y desde el punto de vista de su ecología trófica tenemos a Medina y Arancibia (2002) y el más actualizado corresponde a Alegre et al. (2013) quienes analizaron la variabilidad espacio-temporal de la dieta del jurel entre los años 1977 y 2011 y su respuesta a diferentes variables ambientales permitiendo establecer dos escenarios, uno entre 1977 al 2000 donde la dieta estuvo fuertemente dominada por Euphausiidae y el otro escenario después del año 2000 donde el jurel consumió mayor diversidad de presas pero estando presente también los eufáusidos.

En este sentido, a pesar de la relativa atención dirigida a tener un mayor conocimiento sobre su comportamiento alimenticio del jurel, se considera necesario realizar estudios más detallados sobre su ecología trófica durante años normales y también en años con fuerte influencia del evento El Niño, por lo que el principal objetivo del presente trabajo es analizar la información sobre la dieta y los hábitos alimenticios del T. murphyi en la costa nor-central del mar peruano analizando variaciones cuantitativas en su composición e intensidad alimenticia en relación a factores tales como estacionalidad y tamaño de los ejemplares. En este contexto se evalúa la hipótesis de una ampliación del espectro trófico del jurel durante un evento cálido tan fuerte como se presentó El Niño en el año 1997 en relación a años previos normales.

\section{Materiales y métodos.}

Los ejemplares de jurel fueron colectados directamente de la zona de pesca entre Máncora y Salaverry en la zona norte y frente al Callao en la zona central del Perú (Figura 1) a bordo de embarcaciones pesqueras comerciales equipadas con redes de arrastre semipelágica y de cerco. Las profundidades de captura fluctuaron entre los 20 y $260 \mathrm{~m}$ y el período de estudio estuvo comprendido entre Abril de 1995 y Diciembre de 1997.

Muestras mensuales de jurel se tomaron para su análisis biológico. A cada ejemplar se le midió la longitud total (LT) medidos con una precisión de 0.1 cm y su peso con una precisión de $1 \mathrm{~g}$. Se tomó una muestra estratificada por tallas a fin de asegurar la presencia de ejemplares de todas las tallas en las muestras obtenidas teniendo presente de asegurar un tamaño mínimo de muestra por clase de tallas. Inmediatamente después de la captura, para cada ejemplar se le extrajo el estómago y preservó en formalina al 5\% para prevenir una digestión mayor y se trasladó para su examen en el laboratorio. También se registró el sexo y estadio de madurez sexual.

En el laboratorio el contenido estomacal de cada ejemplar fue analizado y se contabilizaron los

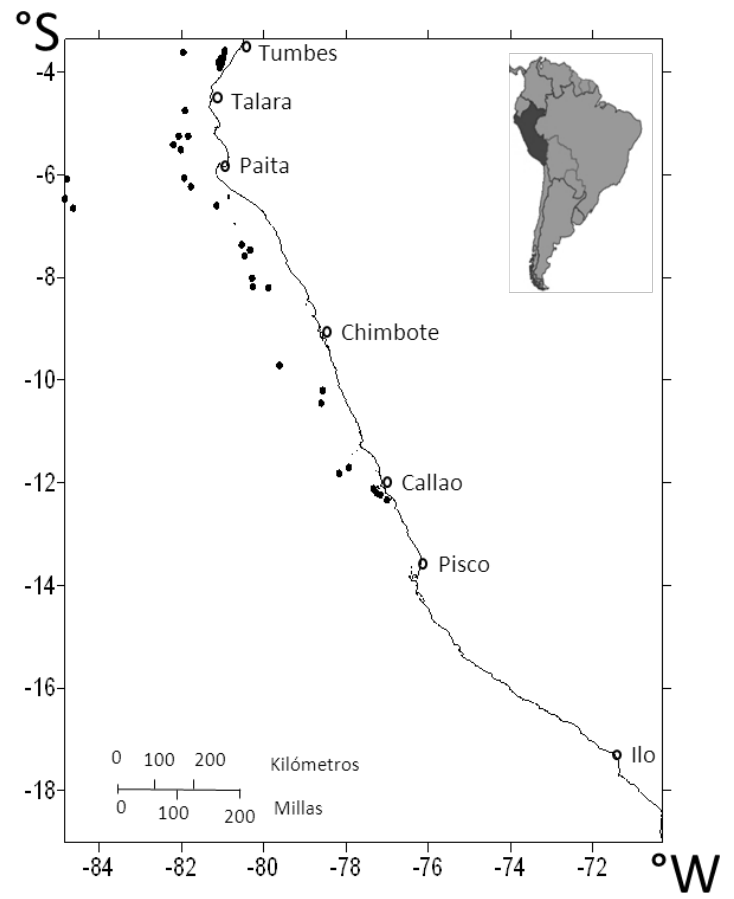

Figura 1. Estaciones de pesca comercial donde se colectaron las muestras del jurel (Trachurus murphyi) durante 1995-1997 en el mar del Perú. 
estómagos vacíos, evertidos y con contenido estomacal. En el caso de los estómagos con alimento, éstos fueron limpiados y secados con papel absorbente para luego registrar el peso del estómago, identificar la presa, contabilizar el número de presas y el peso de las presas con una precisión de $0.01 \mathrm{~g}$. Las presas fueron identificadas en especies o en los casos donde fue posible hasta el menor nivel taxonómico mediante el empleo de literatura especializada tales como claves y listas taxonómicas, todo ello sobre la base de Del Solar \& Alamo (1970) para los crustáceos y Chirichigno (1998) para el caso de los peces.

La Tabla 1 muestra el número de estómagos colectados y analizados por temporada del año entre los años 1995 y 1997, indicando la profundidad media de captura y la zona de pesca. Se alcanzó a analizar un total de 3175 ejemplares.

Con la finalidad de detectar diferencias de los peces, los ejemplares de jurel fueron agrupados en diez grupos de tallas de $4 \mathrm{~cm}$ cada una y los datos fueron posteriormente agrupados por temporadas para estudiar los cambios temporales en sus hábitos alimenticios.

Se realizaron análisis de distribución de frecuencias de los estómagos con alimento en relación al número de ejemplares examinados para el periodo total de estudio; igualmente se determinó la distribución del número ejemplares con estómagos vacíos, evertidos y especialmente con alimento analizando su variación por grupos de tallas y por temporada para los años de estudio. Se estimó el porcentaje de la masa $(\% M)$ de cada una de las presas el estómago y de acuerdo a esto para el análisis de la composición de las dietas los taxa de las principales presas fueron agrupados en categorías de acuerdo a su importancia (Santic et al., 2003; Silva, 1999). Se realizó también el análisis del número de presas predominantes por año.

Los cambios en las dietas anuales por clases de longitud del jurel fueron también analizados, donde los principales grupos alimenticios estuvieron expresados en términos de peso como un porcentaje ontogenéticas en la dieta relacionados con el tamaño consumidas, expresada como la masa (g) de cada presa en relación a la masa total de todas las presas en

del total de las presas en cada grupo de longitud. Se evaluó las diferencias del principal ítem alimenticio por grupos de tallas para los tres años de muestreo e igualmente se analizó por temporada los pesos promedios de los contenidos estomacales por grupos de longitud del jurel. Las diferencias estadísticas ( $\mathrm{p}<$ 0.05) en la composición de la dieta con respecto al tamaño y temporada se evaluó con el test de chicuadrado (Sokal \& Rolf, 1981) aplicado sobre el porcentaje de los contenidos estomacales.

Numerosos Índices han sido descritos para expresar cuantitativamente la importancia de las diferentes presas en la dieta de los peces (Berg, 1979; Hyslop, 1980). En este estudio para conocer la contribución de cada categoría de la presa a la dieta del jurel por temporadas se determinaron dos medidas relativas de las cantidades de presas: 1971)

Índice de Importancia Relativa (IIR) (Pinkas et al.,

donde:

$$
\mathrm{IIR}=(\mathrm{Cn}+\mathrm{Cw}) \mathrm{F}(1)
$$

F: porcentaje de frecuencia de ocurrencia.

Cn: porcentaje de composición numérica.

Cw: porcentaje de composición gravimétrica.

Para calcular la jerarquía de las presas en la dieta se aplicó al valor de IIR el índice de jerarquización (DJ) que toma el valor más alto del IIR y calcula el porcentaje de todos los demás valores a partir de él. Si el porcentaje del ítem presa se encuentra incluido entre el $100 \%$ y $75 \%$ se le considera fundamental; si se ubica entre el $75 \%$ y $50 \%$ se le considera secundario, si está entre el 50\% y 25\% es accesorio, y si está por debajo del 25\% se le considera accidental (Martori, 1991).

Índice de Plenitud Estomacal (Fs) (Letourneur et al., 1997)

$$
(\mathrm{Fs})=100 \mathrm{x}(\mathrm{fw} / \mathrm{f})
$$

donde:

fw : peso del contenido estomacal de cada pez $\mathrm{f}$ (en g).

f : longitud total del pez $\mathrm{f}$ (en cm).

Con la finalidad de identificar patrones en los datos de contenido Tabla 1. Número total de estómagos (n) por temporada para los años colectados entre

\begin{tabular}{|c|c|c|c|c|c|c|c|c|c|}
\hline \multirow[b]{2}{*}{$\begin{array}{l}\text { Estación } \\
\text { del año }\end{array}$} & \multicolumn{3}{|c|}{1995} & \multicolumn{3}{|c|}{1996} & \multicolumn{3}{|c|}{1997} \\
\hline & $\mathrm{n}$ & $\begin{array}{l}\text { Prof. } \\
\text { (m) }\end{array}$ & Zona & $\mathrm{n}$ & $\begin{array}{l}\text { Prof. } \\
\text { (m) }\end{array}$ & Zona & $\mathrm{n}$ & $\begin{array}{l}\text { Prof. } \\
\text { (m) }\end{array}$ & Zona \\
\hline Verano & - & - & - & 575 & $23 / 150$ & $\begin{array}{c}\text { Callao/ } \\
\text { Norte }\end{array}$ & 193 & $2 / 76$ & $\begin{array}{l}\text { Callao } \\
\text { /Norte }\end{array}$ \\
\hline Otoño & 95 & 32 & Norte & 400 & $16 / 161$ & Callao/ & 256 & 80 & Norte \\
\hline Invierno & 80 & 27 & Norte & 420 & $26 / 168$ & $\begin{array}{l}\text { Callao/ } \\
\text { Norte }\end{array}$ & 249 & $31 / 308$ & Norte \\
\hline Primavera & 199 & $16 / 210$ & $\begin{array}{l}\text { Callao } \\
\text { /Norte }\end{array}$ & 397 & $19 / 124$ & $\begin{array}{c}\text { Callao/ } \\
\text { Norte }\end{array}$ & 311 & $32 / 208$ & $\begin{array}{l}\text { Callao } \\
\text { /Norte }\end{array}$ \\
\hline TOTAL & 374 & & & 1792 & & & 1009 & & \\
\hline
\end{tabular}
1995 y 1997. usaron técnicas multivariadas no paramétricas. Para ello se usó el análisis cluster y se construyeron dendrogramas de similaridad usando el coeficiente Bray-Curtis (Bray \& Curtis, 1957) a 
datos pareados aplicado a la raíz cuadrada de los datos de pesos de presas. Los pesos de presas fueron usados porque esta medida refleja mejor la importancia energética de la presa en la dieta que la frecuencia de ocurrencia o abundancia numérica. El análisis cluster fue aplicado a los datos de presas agrupados por años y por grupos de talla del predador con intervalos de 4 cm y por año usando el algoritmo UPGMA (unweigthed pairgroup with arithmetic mean). El análisis multivariado fue realizado con el programa Plymouth Routines in Multivariate Ecological Research (PRIMER, versión 6.0; PRIMER-E Ltd, Plymouth, UK).

\section{Resultados y discusión.}

Intensidad de alimentación.

Se midieron y examinaron los estómagos de 3175 ejemplares de jurel cuyas longitudes totales fluctuaron entre los 22 y $59 \mathrm{~cm}$ con la mayor concentración de ejemplares entre los 26 y $46 \mathrm{~cm}$, a diferencia de los resultados encontrados por el IMARPE en el 2013 cuya estructura de tallas registradas para el primer semestre de ese año osciló entre 22 y $47 \mathrm{~cm}$ y en octubre del mismo año entre 23 y $42 \mathrm{~cm}$. Del total de ejemplares muestreados 2580 (81.2\%) estuvieron con alimento (Figura 2), a diferencia de lo encontrado por Alegre et al., 2013 que analizando más de 20 mil estómagos en un periodo de 34 años el porcentaje de estómagos con contenido en sus estómagos fue de $41.3 \%$, lo que obedece sin duda a una serie de factores que van desde las condiciones biológicas predominantes durante los periodos de estudio que habría que profundizar hasta las diferentes condiciones de muestreo realizadas durante las operaciones de pesca. Realizando un análisis general, es notorio observar la presencia de peces con un amplio rango de tamaños para todo el periodo de estudio, sin embargo, se puede distinguir con claridad un menor número de individuos mayores de $50 \mathrm{~cm}$; Igualmente, se debe resaltar que el porcentaje de ejemplares menores a $31 \mathrm{~cm}$ (talla mínima legal de captura) llega al $16.8 \%$ por lo que el mayor porcentaje

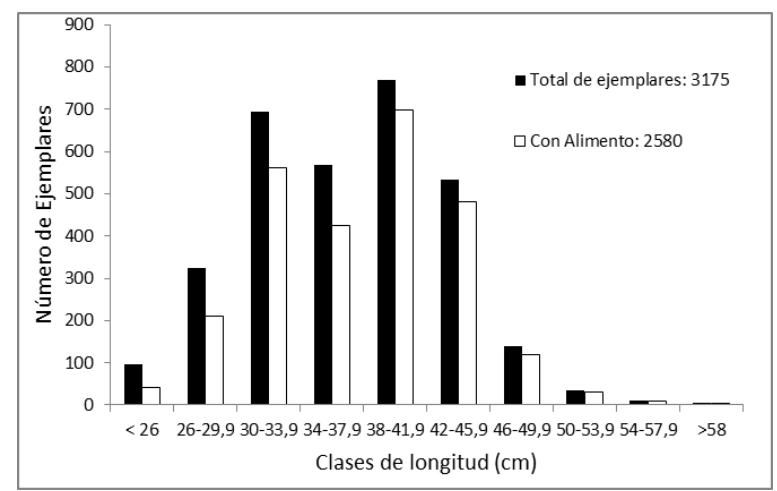

Figura 2. Número total de ejemplares colectados y número de ejemplares cuyos estómagos presentan alimento por clases de longitud. de ejemplares capturados fue predominantemente adulto.

La distribución del número de ejemplares agrupados por clases de longitud cuyos estómagos se presentaban con alimento, vacíos y evertidos se muestra en la Figura 3, donde se aprecia una alta predominancia de estómagos con alimento. El número de estómagos vacíos es notoriamente más bajo en la mayoría de las clases de longitud, siendo en los ejemplares pequeños $(<26 \mathrm{~cm})$ el único grupo de longitud en donde el número de estómagos vacíos supera al número de estómagos con alimento. Igualmente se observa una muy escasa presencia de estómagos evertidos. No ha sido posible encontrar estudios disponibles hasta el momento sobre este tipo de información.

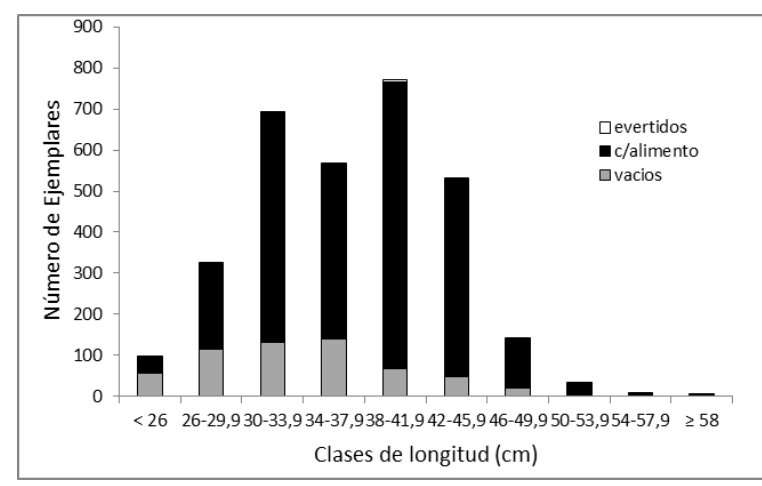

Figura 3. Número de ejemplares de acuerdo a la condición de sus estómagos por clases de longitud.

Analizando estadísticamente la información sólo de los ejemplares con alimento en sus estómagos en términos porcentuales por grupos de tallas (Figura 4), se observa que la proporción de estos estómagos varió significativamente para todos los grupos de tallas de los peces examinados $\left(\chi^{2}=29.6, \mathrm{p}<0.05\right)$, con un máximo de $100 \%$ de contenido estomacal (clase de longitud $54-57.9 \mathrm{~cm}$ ) y un mínimo de $42.3 \%$ (clase de longitud $<26 \mathrm{~cm}$ ).

Examinando la tendencia por temporadas de los ejemplares con alimento en sus estómagos en términos

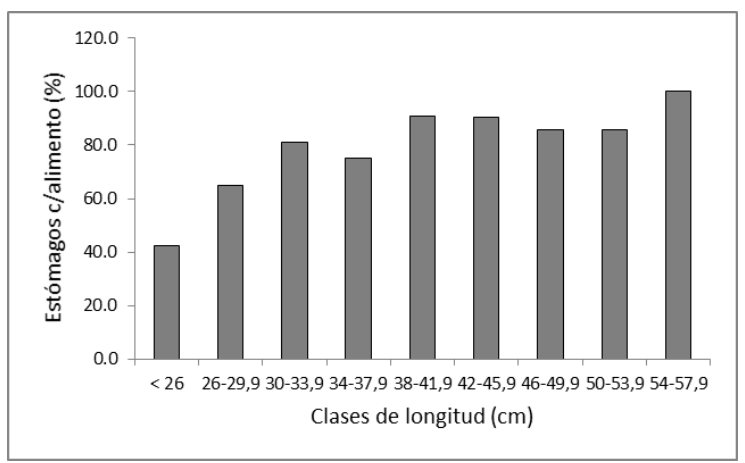

Figura 4. Variación porcentual de los estómagos con alimento del T. murphyi en función al tamaño del cuerpo. 
porcentuales (Figura 5), se observa que predominan los estómagos con alimento en todas las temporadas aunque con mayor relevancia en los años 1996 y 1997, siendo el año 1996 (año previo al evento de El Niño) el mejor año en las condiciones alimenticias con el más alto contenidos estomacal alcanzando un máximo de $93.2 \%$ en otoño de ese año. Analizando estadísticamente esta información para todo el periodo se observa que la proporción de estos estómagos varió significativamente en toda la temporada $\left(\chi^{2}=30.3\right.$, $\mathrm{p}<0.05$ ), pudiéndose interpretar este resultado en el sentido que las diferentes temporadas en el año calendario, por sus condiciones oceanográficas específicas, ofrecen a los peces diferentes condiciones en cuanto a la disponibilidad de alimento.

Composición dietaria del contenido estomacal.

De los contenidos estomacales del jurel, fueron identificados hasta 23 diferentes grupos de presas, entre especies y taxas, pertenecientes a 10 mayores taxas: Teleostei, Euphausiacea, Decapoda, Stomatopoda, Isopoda, Amphipoda, Copepoda, Ostracoda, Cephalopoda y Gasteropoda. La Tabla 2 muestra la composición de la dieta del jurel para cada año y para el periodo total de estudio donde se presentan los principales ítems alimenticios, el peso de las presas identificadas (g), el agrupamiento de presas para el análisis dietario y el \%M para los principales grupos alimenticios.

Para el período total las presas que forman el mayor grupo alimenticio del $T$. murphyi son los crustáceos con el $60.4 \%$ por masa de alimento ingerido seguido por los peces teleósteos con el 39.3 $\%$. Los moluscos constituyen un grupo muy reducido de presas llegando a representar sólo el 0.35\%. En este sentido los grupos de presas más representativos son los crustáceos, entre ellos las especies como Euphausia mucronata (54.3\%) y munidas; y los peces, donde destacan los restos no identificados (35.5\%), los peces identificados (2.35\%) y la especie Odontestes regia (1.44\%). Cabe mencionar la presencia de la sardina en los contenidos estomacales del jurel para los años 1995 y 1996, con una mayor

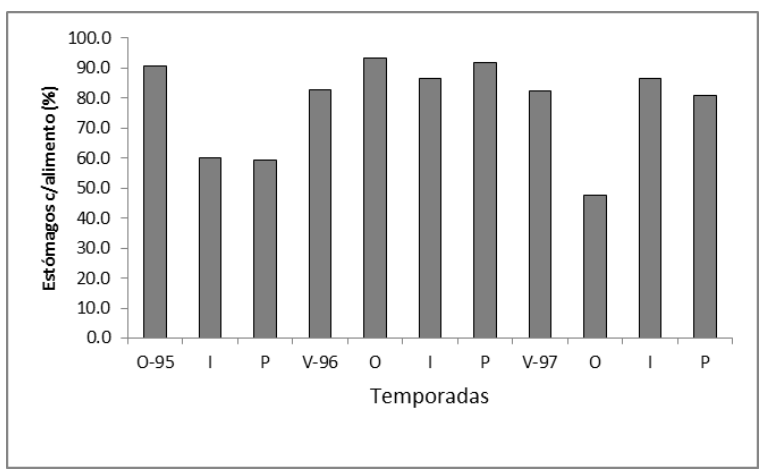

Figura 5. Variación porcentual de los estómagos con alimento del T. murphyi por temporadas. predominancia de peces en general en año 1996, reduciéndose la variedad de peces en 1997, pero aumentando significativamente la presencia de myctófidos. Se observa también la preferencia de una más amplia variedad de crustáceos en el hábito alimenticio del jurel, favorecido posiblemente por el evento de El Niño durante esta temporada.

Estos resultados coinciden en parte con lo señalado por Alamo et al. (1997) quienes encontraron, para el verano e inicios del otoño de 1997 (evento de El Niño) que la composición alimentaria estuvo constituida por crustáceos, peces y moluscos, pero el principal alimento del jurel fueron los crustáceos planctónicos destacando los eufausidos $y$ los peces que constituyeron el segundo grupo en nivel de importancia, destacando la familia Myctophidae y las especies Vicinguerria sp., Leuroglosus urotranus y Triphoturus mexicanus. Los moluscos estuvieron representados por gasterópodos y con bajos valores de IIR por lo que su presencia no tiene mayor importancia en la dieta de esta especie. Sin embargo, de acuerdo al mismo Alamo et al. (1997) en el mes de setiembre de 1997 , en la zona norte, desde los $6^{\circ}$ hasta los $10^{\circ}$ (Chimbote), la dieta del jurel muestra una total ausencia de peces en sus estómagos sólo evidenciando presencia de copépodos y especies de la orden euphausiacea; mientras que desde los $10^{\circ}$ hasta los $18^{\circ}$ (Ilo) hay una gran presencia de peces en su dieta identificándose principalmente a la Vinciguerria sp. y Myctophidae en toda la zona y Triphoturus mexicanus en la zona central.

Igualmente, Alegre et al. (2013) cuando analiza los cambios en la dieta del jurel desde el año 1977 hasta el 2011 observa dos escenarios en la composición de la dieta en términos del porcentaje del peso húmedo, el primero que comenzó en 1977 hasta el año 2000, que comprende el periodo del presente estudio, donde la especie dominante son los eufáusidos (\%W $\leq 96.0)$, después del periodo interdecadal cálido y ENSO 1997-1998, y luego el segundo a partir del 2000 en donde aumentó la proporción de zoeas y P. monodon, indicador de aguas costeras frías (ACF) disminuyendo la presencia de los eufausidos pero sin dejar de ser representativa en su dieta.

Cabe destacar que según Méndez (1981), las especies pelágicas o bentónicas dentro de los carideos, como parte importante de la dieta del jurel según el índice de jerarquización (DJ) sería accidental y se distribuyen en el mar peruano habitando sobre fondos de más de 10-150 m de profundidad sobre el borde del talud y el talud continental y los penaeideos como nectobentónicos se distribuyen sobre los $150 \mathrm{~m}$ en la plataforma continental. Las especies de aguas profundas son las que presentan rangos de distribución geográfica más amplios debido a condiciones más estables de ciertos parámetros como la temperatura y de acuerdo a la distribución geográfica se encuentran 
en la plataforma continental en la zona norte del Perú como su principal hábitat por eso se encuentran mayormente en la dieta alimenticia del jurel.

Estas observaciones se ven reforzadas con los resultados presentados en la misma Tabla 2 donde se muestra el número de especies registradas como presas, ya sea en especies o taxa menor identificada, durante los tres años de estudio, donde se aprecia un aumento del número de especies registradas de sólo 5 en 1995 a 23 en 1997, quedando evidenciado una gran aumento del espectro trófico en el año 1997 que podría estar relacionado con la intrusión de aguas cálidas con una mayor diversidad de la oferta alimenticia (principalmente crustáceos) para el jurel

sobretodo en la zona norte del Perú.

Alimento en relación al tamaño del pez.

La composición dietaria por clases de longitud del jurel para los tres años evaluados en función de \%M de los seis principales grupos de presas se muestran en la Figura 6.

En el año 1995, existe una fuerte preponderancia de consumo de eufáusidos en ejemplares pequeños menores a los $38 \mathrm{~cm}$, para luego reducirse a niveles mínimos de consumo en ejemplares mayores a esta talla. Para este año existe una constante en el consumo de peces con predominancia de restos no identificado de peces (RNI) para ejemplares de jurel en todas las tallas y con mayor presencia en los ejemplares grandes (mayores a $34 \mathrm{~cm}$ )

Tabla 2. Porcentaje de la masa de las presas consumidas por T. murphyi en la costa norte-centro del Perú y el agrupamiento de las principales presas.

\begin{tabular}{|c|c|c|c|c|c|c|c|c|}
\hline \multirow[t]{2}{*}{ Taxon de la presa } & \multirow[t]{2}{*}{ Especies } & \multirow{3}{*}{$\begin{array}{l}\text { Grupo } \\
\text { presas }\end{array}$} & \multicolumn{3}{|c|}{ Peso de las presas (g) } & \multicolumn{2}{|c|}{ Total } & \multirow{3}{*}{$\begin{array}{l}\text { para ejemplares entre } \\
38 \text { y } 46 \mathrm{~cm} \text {. En el año } \\
1996 \text {, la tendencia es } \\
\text { similar que el año }\end{array}$} \\
\hline & & & 1995 & 1996 & 1997 & $\mathrm{~W}(\mathrm{~g})$ & $\% M$ & \\
\hline Crustacea & & & & & & & 2.39 & \\
\hline Euphausiacea & E. mucronata & EUP & 262.3 & 2549.9 & 793.5 & 3605.7 & 54.35 & \\
\hline Decapoda & Munidas sp. & MUN & & 240.3 & & 240.3 & 3.62 & anterior pero con mayor \\
\hline Caridea & & & & 0.3 & 9.0 & 9.3 & & $\begin{array}{l}\text { predominancia en el } \\
\text { consumo de eufáusidos }\end{array}$ \\
\hline Penaeidae & & & & & 0.8 & 0.8 & & $\begin{array}{l}\text { consumo de eurausidos } \\
\text { en todos los grunos de }\end{array}$ \\
\hline Paguridae & & & & & 0.2 & 0.2 & & en todos los grupos de \\
\hline & P. americana & & & & 0.5 & 0.5 & & $\begin{array}{l}\text { tallas, notandose una } \\
\text { mavor preferencia en el }\end{array}$ \\
\hline Stomatopoda & & & & 1.5 & 12.4 & 13.8 & & \\
\hline Amphipoda & S. panamensis & & & & $\begin{array}{r}15.0 \\
0.2\end{array}$ & $\begin{array}{r}15.0 \\
0.2\end{array}$ & & identificados de peces \\
\hline Gammaridea & & & & & 0.2 & 0.2 & & para ejemplares grandes \\
\hline & Phronima sp. & & & & 0.2 & 0.2 & & (> 38 cm). Especímenes \\
\hline Copepoda & & & & & 0.5 & 0.5 & & menores a $42 \quad \mathrm{~cm}$ \\
\hline Calanoida & & & & & 0.3 & 0.3 & & muestran una relativa \\
\hline Isopoda & & & & & 0.03 & 0.03 & & preferencia \\
\hline Ostracoda & & & & & 0.5 & 0.5 & & consumo \\
\hline Otras especies & & & & 14.7 & 37.1 & 51.7 & & crustáceos entre los que \\
\hline Megalopa & & & & & 9.7 & 9.7 & & destacan las múnidas, \\
\hline Zoea & & & & & 55.6 & 55.6 & & carideos \\
\hline Mollusca & & & & & & & & stomatopodos. En el \\
\hline Cephalopoda & & & & & 0.2 & 0.2 & & año 1997 , se mantiene \\
\hline & Loligo sp. & & 11.8 & 2.5 & 8.1 & 22.4 & & la preferencia por los \\
\hline Gasteropoda & & & & & 0.1 & 0.1 & & eufáusidos en todas las \\
\hline Naticidae & & & & & 0.8 & 0.8 & & aunque \\
\hline Pisces & & PEZ & & & & & 2.35 & disminuyendo \\
\hline & Engraulis & & & & & & & preferencia cuando \\
\hline Engraulidae & ringens & & & 5.3 & & 5.3 & & aumenta la longitud de \\
\hline Stromateidae & Trachinotus sp. & & & 10.1 & & 10.1 & & los ejemplares, también \\
\hline Sciaenidae & & & & & 2.7 & 2.7 & & aumenta la preferencia \\
\hline Clupeidae & S. sagax & & 18.3 & 9.0 & & 27.3 & & en el consumo de restos \\
\hline Atherinidae & O. regia & ODO & 53.3 & 42.4 & & 95.7 & 1.44 & no identificados de \\
\hline Belonidae & Belone sp. & & & 46.8 & & 46.8 & & peces en todos los \\
\hline Trichiuridae & & & & & 0.3 & 0.3 & & grupos de tallas siendo \\
\hline Gobiidae & & & & 21.6 & & 21.6 & & más notorio para los \\
\hline Myctophidae & Myctophe & & & 2.0 & 39.5 & 41.5 & & ejemplares mayores \\
\hline No identificados & & RNI & 147.5 & 1633.3 & 573.5 & 2354.3 & 35.49 & $(>42 \mathrm{~cm})$ Igualmente \\
\hline & Número especies & & 5 & 14 & 23 & & & hay una relativa \\
\hline
\end{tabular}


principalmente para ejemplares de talla media (entre 34 y $50 \mathrm{~cm})$.

En este sentido debemos destacar lo observado por Alegre et al. (2013) desde el año 1977 al 2011, respecto a la talla del jurel, donde los individuos menores de $21 \mathrm{~cm}$ la dieta estuvo compuesta mayormente por copepodos $(\% \mathrm{~W}=34.0)$ y coincidiendo en que la proporción de eufáusidos fue aumentando con la talla hasta los $50 \mathrm{~cm}$ y a partir de los $51 \mathrm{~cm}$ fue mayor la ingesta de peces mesopelágicos y otros teleósteos. En general, los eufáusidos y teleósteos son los principales grupos presentes en la dieta en los tres años. Los eufáusidos están presentes en todos los grupos de tallas de los años 95, 96 y 97, sin embargo en el año 95 hay ausencia de eufáusidos en el grupo de talla más grande (46-49.9 cm) siendo reemplazado integramente por restos de peces. Los eufáusidos fueron el grupo de presas más importante para ejemplares de pequeñas y medianas tallas (hasta $42 \mathrm{~cm}$ ); mientras que se aprecia un gradual aumento con la talla del jurel en la
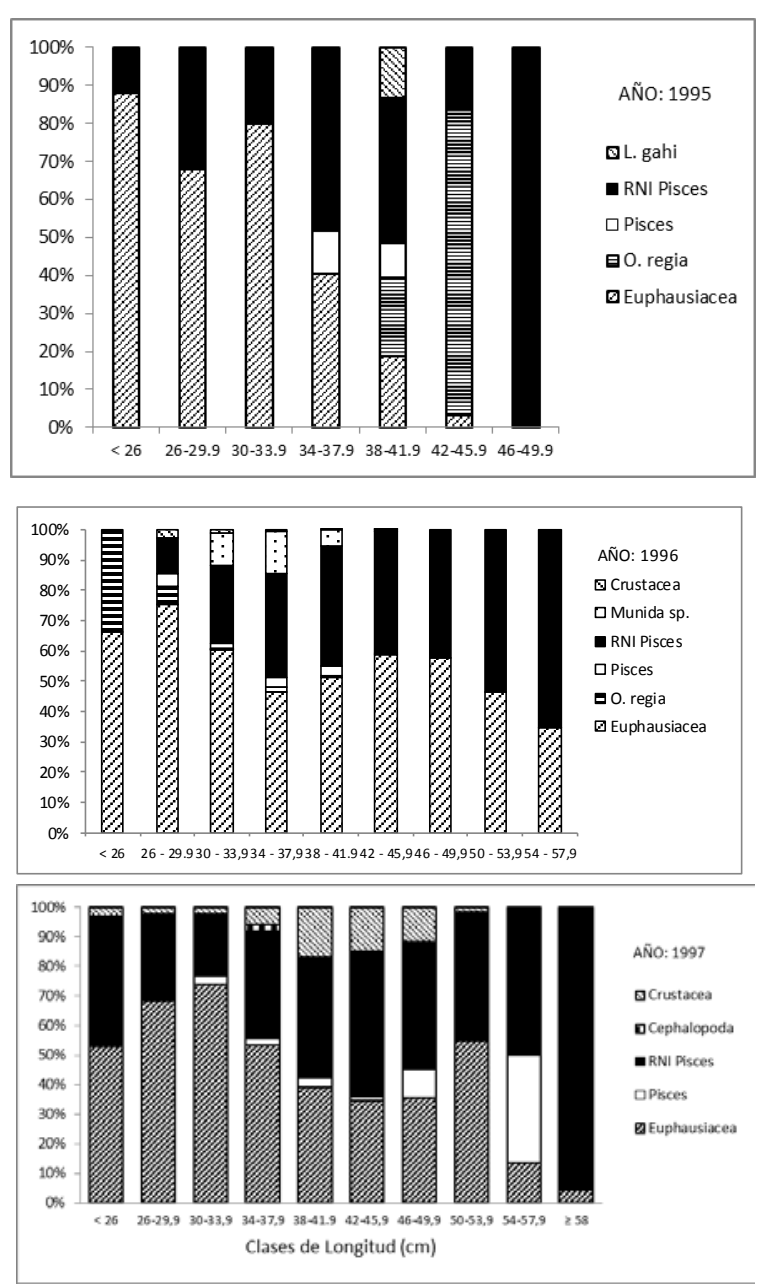

Figura 6. Composición de la dieta (\%) de las principales presas para cada grupo de longitud del T. murphyi entre 1995 y 1997.

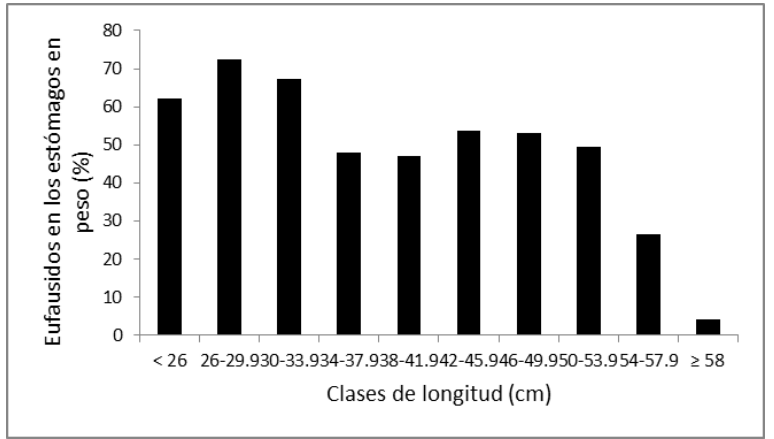

Figura 7. Variación porcentual de la presencia de eufáusidos en peso en los estómagos del $T$. murphyi por clases de longitud.

preferencia como presa por peces teleósteos. Analizando estadísticamente la información la proporción de eufáusidos como principal alimento para todo el periodo de estudio en términos porcentuales (Figura 7) se observa que disminuye significativamente con el incremento de tamaño del pez $\left(\chi^{2}=26.8, p<0.05\right)$. Para cada año en forma individual también se observó este mismo comportamiento, siendo más notorio en el año 1995 $\left(\chi^{2}=189.4, \mathrm{p}<0.05\right)$.

La Figura 8 muestra los resultados del análisis clúster aplicado a los datos de contenido estomacal en relación a la longitud de los individuos. El análisis clúster aplicado para el año 1995 organizó las 8 clases de talla en 2 grupos principales a un nivel de similaridad mayor de $40 \%$ correspondientes a tallas de $<26$ a $49.9 \mathrm{~cm}$ y un grupo representado por la clase de 50 a $53.9 \mathrm{~cm}$. En el caso del año 1996 se observa algo muy similar con dos grupos bien definidos entre las clases de 26 a $49.9 \mathrm{~cm}$ y entre 50 a 57.9. En este año los individuos con longitudes $<26 \mathrm{~cm}$ forman un grupo aislado que no muestran una similitud muy débil con la dieta de las clases antes mencionadas. En 1997 un año con evento de El Niño, los individuos forman dos grupos definidos en relación al contenido estomacal, un grupo de individuos entre $<26$ a 53.9 $\mathrm{cm}$ y el otro entre 54 a $>58 \mathrm{~cm}$. Aun cuando el espectro alimentario del jurel se amplia de manera significativa durante el evento El Niño, el patrón de formación de dos grupos con dieta diferenciada se mantiene. Este patrón se relaciona con la predominancia en la dieta de eufáusidos en la dieta de individuos pertenecientes a las clases de longitud entre 26 y alrededor de $50 \mathrm{~cm}$ y la predominancia de peces en la dieta de individuos $>50 \mathrm{~cm}$. Este patrón de agrupamiento concuerda con los resultados obtenidos por Alegre et al. (2013).

Variación estacional en la composición de la dieta.

Los resultados proporcionados por el análisis del Indice de Importancia relativa (IIR) y el Indice de Jerarquización se muestran en el Anexo 1. De acuerdo a los análisis del Indice de Jerarquización se considera como presas fundamentales a los eufáusidos y a los 

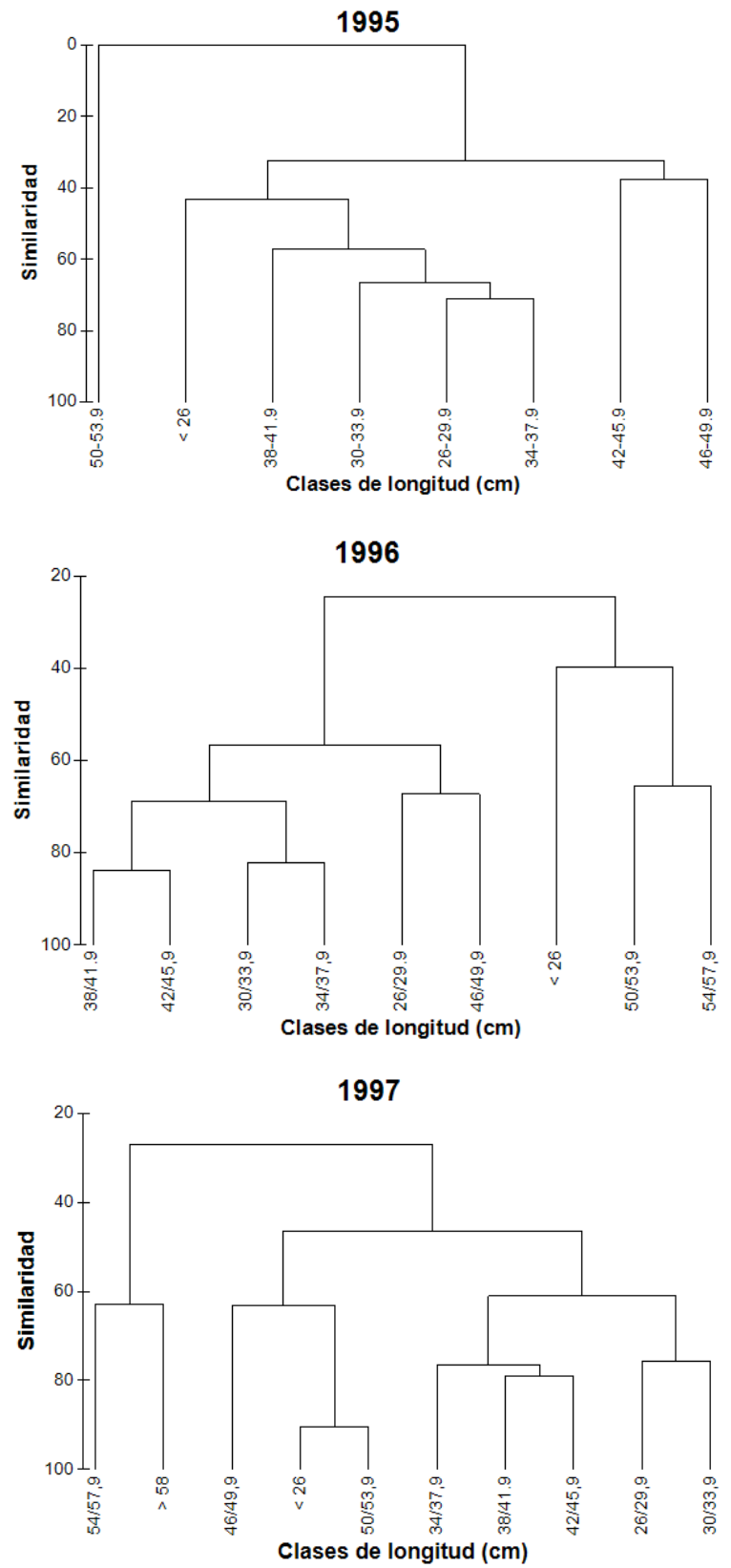

Figura 8. Dendrogramas de similaridad (\%) de Bray-Curtis de la composición de la dieta de $T$. murphyi de diferentes grupos de talla.

restos no identificados de peces (RNI), luego se puede señalar como presas secundarias a otras especies de crustáceos (copepodos y S. panamensis) y como presas accidentales a otras especies de peces identificados.

La Figura 9 presenta la variación interanual del IIR para los cuatro principales grupos de presas en la dieta del jurel, donde los eufausidos tienen una presencia constante durante todo el periodo, lo mismo sucede con los RNI pero con una clara disminución en en la temporada de invierno de 1995 . Se destaca la notable preferencia de la especie E. mucronata en los años
1995 y 1996 pero diversificándose en 1997 con otras especies de eufáusidos. Igualmente se observa esta diversificación en 1997 con la aparición importante de otros crustáceos pequeños como copépodos y stomatópodos (S. panamensis). Los restos identificados de peces con bajos valores del IIR muestran una relativa e intermitente presencia, entre los que destacan especies de las familias Gobiidae, Belonidae, Myctophidae y las especie $O$. regia, destacando los mictófidos durante todo el año 1997. Otros crustáceos tambien con valores bajos del IIR sólo muestran presencia en la dieta del jurel en el año 1997 descendiendo el valor IIR en el invierno de este periodo.

En este sentido, estos resultados muestran una cierta concordancia con lo hallado por Imarpe (2009), señalando que el jurel presenta un amplio espectro trófico conformado por crustáceos, teleósteos, moluscos, urocordados y poliquetos sobretodo de peces en la zona central y con un comportamiento predador más intenso encontrándose sobre el tercer nivel trófico. La mayor parte de especies de eufáusidos se asocia a la cercanía al frente ecuatorial en los focos de surgencia, mientras que en el sur fuera de la costa predominó la especie E. mucronata. De acuerdo al IIR es de manifiesto predominio entre los crustáceos especies de la orden Euphausiacea, siguiéndoles en presencia las larvas zoeas y otros tipos de pequeños crustáceos que llegan a ser incluso predominantes al finalizar el año. Entre los peces es la anchoveta el principal alimento determinando su marcada preferencia.

De acuerdo Alamo et al. (1997), los valores de IIR señalan que especies de la orden Euphausiacea presentan predominancia en la dieta del jurel en toda la zona central y norte del Perú, siguiéndole en importancia los copépodos. Medina y Arancibia (2002) para el caso del jurel frente a la costa norte de Chile señalan que para los años 1993-1994 los eufáusidos también fueron las principales presas en todas las estaciones del año, destacando también el pez mesopelágico Vinciguerria sp. De igual manera cabe también destacar la presencia importante de

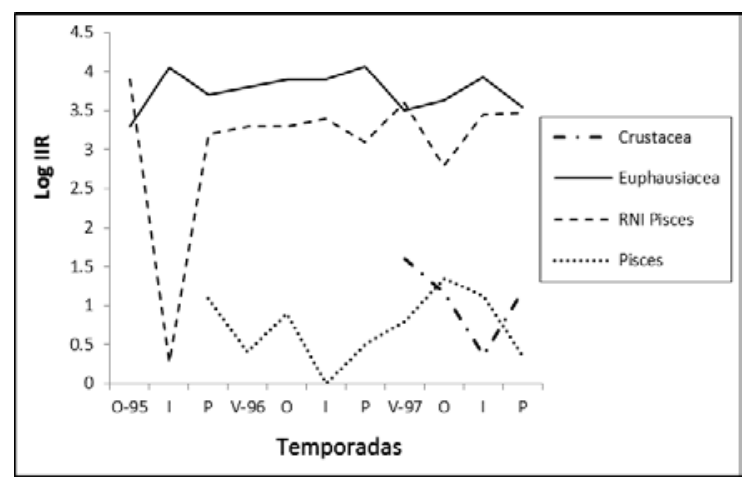

Figura 9. Variación interanual del IIR para los principales grupos de presas del jurel 1995-1997. 


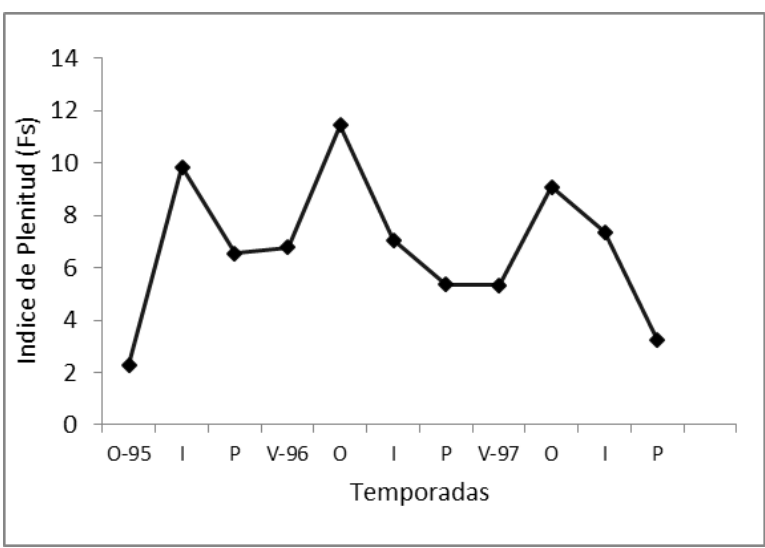

Figura 10. Variación interanual del Indice de Plenitud del estómago (Fs) del Jurel 1995-1997.

ejemplares grandes de Engraulis ringens en los estómagos del jurel en esa zona del Pacífico.

El Indice de plenitud estomacal (Fs) para el periodo total de estudio (Figura 10) muestra un comportamiento oscilante y variable destacando los máximos valores en la temporada de invierno para el año 1995, mientras que en los años 1996 y 1997 es la temporada de otoño la que sobresale con los máximos valores. Durante el año 1997 se presenta una disminución en los valores de Fs hasta fines de ese año reflejando escasas condiciones de oferta alimenticia que aunque se diversificó en el espectro alimentario la oferta disminuyó en cantidad lo que se refleja en el Indice de plenitud probablemente asociado a los cambios de las condiciones oceanograficas que se presentaron en las etapas tempranas del evento de El Niño excepcional 19971998. En este sentido los resultados coinciden con el Indice de llenura (\%) en relación a la proporción de presas en los estómagos del jurel señalado por Alegre et al. (2013) que tuvo un comportamiento variable en todo el periodo de estudio.

\section{Conclusiones.}

1. Las presas que forman el mayor grupo alimenticio del Trachurus murphyi son los crustáceos, representando el $60.4 \%$ del peso del contenido estomacal, seguido por los peces teleósteos con el $39.3 \%$ y los moluscos que sólo constituyen el $0.35 \%$.

2. Entre los crustáceos la especie E. mucronata representa el principal ítem alimenticio con el 54.3\%. Los peces alcanzan un porcentaje de $35.5 \%$ aunque sin poder identificar la especie predominante por el estado de descomposición de los contenidos estomacales. Es destacable la presencia de la especie $O$. regia en el año 1996 y mictófidos durante todo el año 1997.

3. El número de especies de presas identificadas en los tres años aumenta en el tiempo registrándose sólo 5 especies en 1995 y luego alcanzar 23 especies en 1997, evidenciándose así un aumento en el espectro alimentario por la ocurrencia del evento El Niño 1997/98 y reafirmando su condición de predador oportunista en el ecosistema pelágico-oceánico del mar peruano.

4. En 1995 se encontró una fuerte preponderancia de consumo de eufáusidos para ejemplares pequeños de jurel menores a los $38 \mathrm{~cm}$., disminuyendo luego su consumo para ejemplares de mayor talla. El consumo de peces tiene un comportamiento contrario aumentando su consumo para los ejemplares de mayor tamaño. En 1996 el comportamiento alimenticio es similar que en 1995, pero con mayor predominancia en el consumo de eufáusidos en todos los grupos de tallas.

5. En 1997, durante el evento de El Niño, se mantiene la preferencia de eufáusidos en todas las tallas aunque en menor intensidad, aumentando en forma relativa la preferencia de otros crustáceos para ejemplares de talla media evidenciando un cambio en el comportamiento alimentario del jurel durante este evento.

El análisis cluster muestra la conformación de dos grupos en relación a la talla similar para los 3 años de estudio ( 26 a 50 y >50). Este patrón se relaciona con la predominancia en la dieta de eufáusidos en la dieta de individuos pertenecientes a las clases de longitud entre 26 y alrededor de $50 \mathrm{~cm}$ y la predominancia de peces en la dieta de individuos $>50 \mathrm{~cm}$.

\section{Agradecimientos.}

Se agradece al Instituto Alfred Wegener (AWI) de Alemania y al Dr. Wolf Arnzt por el apoyo recibido en el financiamiento de la presente investigación como parte del proyecto: "Predation of Peruvian hake and jack mackerel on the south pacific off the Peruvian coast”. Igualmente un agradecimiento especial al Ing. Luis Garayar Meléndez† por su colaboración en los muestreos a bordo de las embarcaciones.

\section{Literatura citada.}

Alamo A., Espinoza P., Zubieta P., \& Navarro I. 1997. Comportamiento dietario de los principales recursos pelágicos peruanos en verano y comienzos de otoño 1997. Inf. IMARPE 127: 82-89.

Alegre A., Espinoza P. \& Espino M. 2013. Ecología trófica del jurel Trachurus murphyi en el Perú entre 1977 2011. En: Cirske J., R. Guevara-Carrasco \& M. Espino (Eds). Ecología, Pesquería y Conservación del jurel (Trachurus murphyi) en el Perú. Rev. peru biol. Número especial 20(1): 075-082 (setiembre 2013).

Bray J R. \& Curtis J.T. 1957. An ordination of the upland forest communities of southern Wisconsin. Ecological Monographs, 27, 325 - 349.

Berg J. 1979. Discussion of the methods of investigating the food of fishes with reference to a preliminary study of the food of Gobiusculus flavescens (Gobidae). Mar. Biol., 50: 263-273.

Berry F. \& Cohen L. 1974. Synopsis of Trachurus. Quarterly Journal of the Florida Academy of Sciences. 35(4) 1972. 
Chirichigno N. 1998. Clave para identificar los peces marinos del Perú. Colaborador J. Vélez. Instituto del Mar del Perú. Publicación especial 2da ed. Revisada y actualizada.496 pp.

Del Solar E. \& Alamo V. 1970. Exploración Sobre Distribución de Langostinos y Otros Crustáceos de la Zona Norte. Inform. Esp. Cruceros SNP-1 7009 (Agosto-septiembre de 1970).

Dioses T. 2013. Patrones de distribución y abundancia del jurel Trachurus murphyi en el Perú. En: Csirke J., R. Guevara-Carrasco \& M. Espino (Eds). Ecología, Pesquería y conservación del jurel (Trachurus murphyi) en el Perú. Rev. Peru.biol. número especial 20(1):067074 (Septiembre 2013).

Espino M. 2013. El jurel Trachurus murphyi y las variables ambientales de macroescala. En: Csirke J., R. GuevaraCarrasco \& M. Espino (Eds). Ecología, Pesquería y conservación del jurel (Trachurus murphyi) en el Perú. Rev. Peru.biol. número especial 20(1):009-020 (Septiembre 2013).

Flores R., Espino M., Luque G. \& J. Quispe. 2013. Patrones de variabilidad ambiental en el mar peruano. En: Csirke J., R. Guevara-Carrasco \& M. Espino (Eds). Ecología, Pesquería y conservación del jurel (Trachurus murphyi) en el Perú. Rev. Peru.biol. número especial 20(1):021028 (Septiembre 2013).

Hyslop E. 1980. Stomach contents analysis - A review of methods and their application. J. Fish. Biol., 17: 411429.

Imarpe. 2009. Prospección Bioceanográfica de los recursos jurel y caballa - 2009. Convenio Produce-Imarpe.

Imarpe. 2013. Desarrollo de la Pesquería del jurel durante el 2013, situación actual del recurso y perspectivas de explotación para el 2014. Informe Interno. Callao Diciembre, 2013.

Letourneur Y., Galzin R. \& Harmelin-Vivien M. 1997. Temporal variations in the diet of the damselfish Stegastes nigricans (Lacepede) on a Reunion fringing reef. J. Exp. Mar. Biol. Ecol. 217 (1997) 1- 18.
Martori A. 1991. Alimentación de los adultos de Eoproctus asper (Dugés 1853) en la montaña media del Pirineo catalán. (España). Rev. Esp. Herp. 5:23-36.

Medina M. \& Arancibia H. 2002. Dinámica trófica del jurel (Trachurus symmetricus murphyi) en el norte de chile. Inves. Mar. Valparaiso 30(1): 45-55, 2002.

Méndez M. (1981). Claves y Distribución de langostinos y camarones. Imarpe. Vol 5.

Ñiquen M., M. Bouchon, D.Ulloa \& A. Medina. 2013. Análisis de la Pesquería del jurel en el Perú. En: Csirke J., R. Guevara-Carrasco \& M. Espino (Eds). Ecología, Pesquería y conservación del jurel (Trachurus murphyi) en el Perú. Rev.Peru.biol. número especial 20(1):097106 (Septiembre 2013).

Pastor E. 1994. El recurso jurel (Trachurus murphyi) especie de alto valor comercial. Revista de Circulación Mundial Pesca. Volumen 62.

Pinkas L., Oliphant M. \& Iverson Z. 1971. Food habitat of albacore bluefin, tuna and bonito in California waters, California Department of Fish and Game. Fish Bulletin 152: 1-105.

Sanchez J., A. Perea, B. Buitrón \& L. Romero. 2013. Escala de madurez gonadal del jurel Trachurus murphyi Nichol 1920. En: Csirke J., R. Guevara-Carrasco \& M. Espino (Eds). Ecología, Pesquería y conservación del jurel (Trachurus murphyi) en el Perú. Rev.Peru.biol. número especial 20(1):035-044 (Septiembre 2013).

Santic M., Jardas I. \& Pallaoro A. 2003. Feeding habits of Mediterranean horse mackerel, Trachurus mediterraneus (Carangidae), in the Central Adriatic Sea. Cybium 2003, 27(4): 247-253.

Silva A. 1999. Feedings habits of John Dory, Zeus faber, off the Portuguese continental coast. J. Mar. Biol. Ass U.K. 79: $333-340$.

Sokal R. \& Rolf F. 1981. Biometry: The principles and practices of statistics in biological Research. 636 p. San Francisco: Freemen.

Subsecretaría de Pesca. 2000. Cuota Global de Captura para la pesquería del recurso jurel año 2001. Informe Técnico (R. Pesq.) No 70. 
Anexo 1. Índice de Importancia Relativa (Log IIR) y el Índice de Jerarquización (DJ) para el Jurel

\begin{tabular}{|c|c|c|c|c|c|c|c|c|c|c|c|c|c|c|}
\hline & \multicolumn{3}{|c|}{1995} & \multicolumn{4}{|c|}{1996} & \multicolumn{4}{|c|}{1997} & \multirow{2}{*}{\begin{tabular}{|c|} 
IIR \\
Prom.
\end{tabular}} & \multirow[t]{2}{*}{$\%$ IIR } & \multirow[t]{2}{*}{ DJ } \\
\hline & Ото & INV & PRI & VER & Ото & INV & PRI & VER & Ото & INV & PRI & & & \\
\hline \multicolumn{15}{|l|}{ CRUSTACEA: } \\
\hline Crustacea & & & & -0.2 & & & -0.28 & 2.1 & 1.17 & -0.9 & 0.87 & 0.46 & 5.33 & 12.0 \\
\hline Ostracoda & & & & & & & & 0.57 & & -0.4 & \begin{tabular}{|l|}
-2.05 \\
\end{tabular} & -0.64 & -7.44 & -16.7 \\
\hline Copepoda nd. & & & & & & & & 2.78 & & & & 2.78 & 32.48 & 72.8 \\
\hline Stomatopoda & & & & & & & -0.9 & 2.01 & -0.79 & 0.36 & & 0.17 & 1.99 & 4.5 \\
\hline Isopoda & & & & & & & & -1.2 & & & & -1.22 & -14.25 & -31.9 \\
\hline Calanoida & & & & & & & & 1.72 & & & -2.05 & -0.17 & -1.93 & -4.3 \\
\hline Amphipoda & & & & & & & & 0.67 & & & & 0.67 & 7.83 & 17.5 \\
\hline Caridea & & & & & & & -1.6 & 1.7 & & & & 0.05 & 0.58 & 1.3 \\
\hline Paguridae & & & & & & & & -0.9 & & -2 & -0.13 & -1.02 & -11.88 & -26.6 \\
\hline Gammaridea & & & & & & & & 0.59 & & & & 0.59 & 6.89 & 15.4 \\
\hline Euphausiacea & & & & & & & & 3.5 & 3.64 & 3.93 & 3.55 & 3.66 & 42.70 & 95.7 \\
\hline E. mucronata & 3.3 & 4.06 & 3.7 & 3.8 & 3.9 & 3.9 & 4.07 & & & & & 3.819 & 44.61 & 100.0 \\
\hline Munida & & & & 1.5 & 1.9 & 0.5 & & & & & & 1.30 & 15.19 & 34.0 \\
\hline P. americana & & & & & & & & & -2.3 & -0.2 & & -1.23 & -14.37 & -32.2 \\
\hline S. panamensis & & & & & & & & & & & 2.17 & 2.17 & 25.35 & 56.8 \\
\hline Penaeidae & & & & & & & & & & & -1.42 & -1.42 & -16.59 & -37.2 \\
\hline Megalopa & & & & & & & -2.4 & 2.17 & & -1.1 & 1.95 & 0.16 & 1.84 & 4.1 \\
\hline \multirow[t]{2}{*}{ Zoeas } & & & & & -1.2 & & & 0.6 & & -0.2 & 0.86 & 0.01 & 0.06 & 0.1 \\
\hline & & & & & & & & & & & & & 0.00 & \\
\hline TELEOSTOMI: & & & & & & & & & & & & & 0.00 & \\
\hline RNI & 3.9 & 0.27 & 3.2 & 3.3 & 3.3 & 3.4 & 3.1 & 3.6 & 2.79 & 3.45 & 3.48 & 3.07 & 35.89 & 80.4 \\
\hline Myctophidae & & & & & & -1.4 & & 0.8 & 1.34 & 1.12 & 0.35 & 0.44 & 5.16 & 11.6 \\
\hline Gobiidae & & & & & & & 0.5 & & & & & 0.50 & 5.84 & 13.1 \\
\hline Trichiuridae & & & & & & & & & -1.52 & & & -1.52 & -17.76 & -39.8 \\
\hline Sciaenidae & & & & & & & & & & & -0.49 & -0.49 & -5.72 & -12.8 \\
\hline S. sagax sagax & & & 0.8 & -0.2 & & & & & & & & 0.33 & 3.80 & 8.5 \\
\hline O. regia regia & & & 1.4 & 0.4 & -0.2 & & -0.58 & & & & & 0.26 & 2.98 & 6.7 \\
\hline Belone sp. & & & & & 0.9 & & & & & & & 0.90 & 10.51 & 23.6 \\
\hline Engraulis ringens & & & & & -1.1 & & & & & & & -1.10 & -12.85 & -28.8 \\
\hline Peprilus medius & & & & & & -0.6 & & & & & & -0.60 & -7.01 & -15.7 \\
\hline \multirow[t]{2}{*}{ Larvas } & & & & & & & & & & & -0.54 & -0.54 & -6.31 & -14.1 \\
\hline & & & & & & & & & & & & & 0.00 & \\
\hline MOLLUSCA : & & & & & & & & & & & & & 0.00 & \\
\hline Naticidae & & & & & & & & 0.19 & & 0.65 & & 0.42 & 4.91 & 11.0 \\
\hline Cephalopoda & & & & & & & & -1.2 & -1.69 & & & -1.42 & -16.59 & -37.2 \\
\hline Gasteropoda & & & & & & & & & & & -1.43 & -1.43 & -16.71 & -37.4 \\
\hline Loligo gahi & & & 0.3 & & -1.4 & & & & & & $-0.07 \mid$ & -0.39 & -4.56 & -10.2 \\
\hline
\end{tabular}

${ }^{1}$ Facultad de Pesquería, Universidad Nacional Agraria La Molina, Apdo. 12-056, Lima 100-Perú, horrego@lamolina.edu.pe.

2 Facultad de Pesquería, Universidad Nacional Agraria La Molina, Apdo. 12-056, Lima 100-Perú, jmendo@lamolina.edu.pe. 